\title{
Seguridad en Anestesia. Un checklist entre los Anestesiólogos en Chile
}

\section{Safety in Anesthesia. A checklist of Anesthesiologists in Chile}

\author{
CRISTIÁN ROCCO M. MD. ${ }^{1}$, MICHAEL SOTO M. ${ }^{3}$, MATÍAS FEIJÓO P. ${ }^{3}$, WALDO MERINO U. MD. ${ }^{2,3}$
}

\begin{abstract}
Aim: To describe the knowledge of the country's anesthesiologists about perioperative safety protocols in order to contribute information to the discussion of a general system for the registration and analysis of incidents as part of the implementation of the Helsinki Declaration on Patient Safety in Chile. Materials and Methods: The information was obtained through 100 surveys sent anonymously to members of the Chilean Society of Anesthesiology, which were answered voluntarily. Results: 89 anesthesiologists responded to the survey. 30\% are familiar with the Helsinki Declaration. The most established protocols in their clinical centers were infection control (78\%), malignant hyperthermia (72\%) and drug monitoring (70\%). By contrast, the protocols for massive hemorrhage, local anesthetic toxicity and anaphylaxis are observed to a lesser degree. The inclusion of an annual report on mortality and morbidity was recorded in $35 \%$ and an annual report on measures taken and outcomes obtained in the improvement of patient safety was only $23 \%$. Conclusions: A low level of knowledge of the Helsinki Declaration is found among the anesthesiologists of the SACH. Not all its protocols are widely implemented, which makes it necessary to develop a policy to assess the development and impact of this Declaration in Chile. It is urgent to strengthen a national registration system to correct behaviors and thus strengthen perioperative safety to the benefit of our patients.
\end{abstract}

Key words: Anesthesia, Safety, Perioperative care, Complications.

\footnotetext{
Anestesiólogo, Clínica Las Condes, Santiago.

Anestesiólogo, Hospital Doctor Hernán Henríquez Aravena, Temuco.

Facultad de Medicina, Universidad de La Frontera, Temuco.
}

Fecha de recepción: 01 de marzo de 2018

Fecha de aceptación : 28 de marzo de 2018

\section{ORCID}

https://orcid.org/0000-0002-9315-6973

\section{Correspondencia:}

Waldo Merino Urrutia

Los Pablos 2860 casa 45.

Email: wamerino@gmail.com

+56974954831 


\begin{abstract}
Resumen
Objetivo: Describir el conocimiento de los anestesiólogos del país acerca de los protocolos de seguridad perioperatorios, para aportar información en la discusión de un sistema general de registro y análisis de incidentes, en el marco de la implementación de la declaración de Helsinki sobre la seguridad del paciente en nuestro país. Material y Método: La información se obtuvo a través de 100 encuestas enviadas de anónimamente a miembros de la sociedad chilena de anestesiología, la que fue respondida de forma voluntaria. Resultados: Contestaron la encuesta 89 anestesiólogos. Un 30\% conoce la Declaración de Helsinki. Los protocolos más establecidos en sus centros clínicos fueron el control de infecciones (78\%) Hipertermia maligna (72\%) y control de fármacos (70\%). Al contrario, los protocolos de hemorragia masiva, intoxicación por anestésicos locales y anafilaxia se observan en menor porcentaje. La realización de un informe anual de mortalidad y morbilidad se consignó en un 35\% y un reporte anual de medidas tomadas y de resultados obtenidos en la mejora de la seguridad del paciente fue de sólo un $23 \%$. Conclusiones: Se observa un bajo nivel de conocimiento de la declaración de Helsinki entre los anestesiólogos de la SACH. No todos sus protocolos están ampliamente implementados, lo que se hace necesario desarrollar una política de evaluación del desarrollo e impacto de esta declaración en nuestro país. Es imperioso fortalecer un sistema nacional de registro para corregir conductas, y de esta forma fortalecer la seguridad en el perioperatorio en beneficio de nuestros pacientes.
\end{abstract}

\section{Palabras clave:}

Anestesia, Seguridad, Cuidado Perioperatorio, Complicaciones.

\section{Introducción}

a seguridad del paciente en el perioperatorio ha sido siempre un tema fundamental, sin embargo, hoy ha cobrado cada vez mayor importancia, en parte por la relevancia del derecho de estos a estar seguros y por tener un rol activo en su seguridad. Incluso los hospitales de alta eficacia y rendimiento, presentan una tasa de error y resultados adversos que son relativamente altos. El informe "To err is human", del Institute of Medicine de Estados Unidos, publicado el año 1999, calculó en base a estudios epidemiológicos realizados en años previos que entre 44.000 y 98.000 pacientes fallecían en los hospitales norteamericanos como consecuencia de errores sanitarios. Desde entonces se ha generado un especial interés en los procesos y en la implementación de sistemas para mejorar la seguridad de nuestros pacientes. En varios países se ha implementado sistemas de notificación de incidentes, anónimos y voluntarios, cuyo propósito es el registro y análisis sistemático del incidente con el objetivo de aprender del error y cambiar la cultura de seguridad[1].

La anestesiología, que por su desarrollo histórico y su posición estratégica es una especialidad clave, debe liderar el cambio en la cultura de seguridad.

La declaración de Helsinki de "Seguridad del Paciente en Anestesiología", firmada y adoptada en 2010 por la European Board of Anaesthesiology (EBA) y la European Society of Anesthesiology (ESA), y ratificada en el año 2012 por la Confederación Latinoamericana de Sociedades de Anestesiología (CLASA), a la cual pertenece la Sociedad de Anestesiología de Chile $(\mathrm{SACH})$, declara una serie de principios y establece recomendaciones de carácter preventivo para instituciones y departamentos de Anestesiología[2].

Los principales requisitos que deben cumplir las instituciones y departamentos de anestesiología según la declaración de Helsinki son:

Estándares mínimos de monitorización recomendados por EBA (quirófano y Recuperación post anestésica).

Protocolos estándares de sedación.

Implementación del listado de verificación quirúrgica.

Informe anual sobre mejora de la seguridad del paciente que incluya medidas adoptadas y resultados de las mismas.

Informe anual de morbimortalidad asociada a la anestesia. 
Contribución a auditorías clínicas nacionales de prácticas seguras.

Sistema de registro de incidentes críticos.

Otro punto que establece como requisito es que todas las instituciones debieran tener protocolos para el manejo de las siguientes situaciones:[2,3,4,5,7]:

Revisión de equipos y fármacos.

Valoración preoperatoria.

Etiquetado de jeringas.

Control de infecciones.

Alivio del dolor.

Intubación difícil.

Hipertermia maligna.

Anafilaxia.

Toxicidad por anestésicos locales.

Hemorragia masiva.

Actualmente en nuestro país existe mucha variabilidad en el cumplimiento de estas recomendaciones, cuando existen y/o están disponibles, y pese a los esfuerzos, nuestro país aún no cuenta con un organismo dedicado a la seguridad en anestesia, un sistema general de registro y análisis de incidentes, ni menos un sistema de vigilancia epidemiológica de eventos adversos relacionados específicamente con esta especialidad. Aún más, no tenemos antecedentes del conocimiento acerca del protocolo entre las instituciones ni de la aplicación o cumplimiento de este por parte de los profesionales.

El objetivo del presente estudio, a través de una encuesta, es describir el conocimiento de los anestesiólogos del país en los aspectos relacionados con la seguridad del paciente en el perioperatorio.

\section{Material y Métodos}

La información para este estudio fue recogida a través de una encuesta enviada a los miembros de la "Sociedad Chilena de Anestesiología" SACH, en el mes de octubre del 2016. La encuesta consta de 14 preguntas cerradas con las opciones de respuesta $\mathrm{SI}$, NO y dependiendo de la pregunta, se agrega NO SABE o PARCIALMENTE. Esta encuesta fue contestada de forma anónima y voluntaria a través de una invitación enviada por correo electrónico que, de ser aceptada a través de un link, permitía contestar las preguntas a través de la plataforma SurveyMonkey ${ }^{\circledR}$. La muestra se obtuvo a través del envío de 100 invitaciones al azar de un universo de 380 socios activos con mail vigente. Se utilizó Microsoft Excel $2013^{\circledR}$ para análisis de datos.

\section{Resultados}

La encuesta fue contestada por 89 anestesistas de un total de 100 invitaciones enviadas de forma aleatoria y con efectiva recepción del mail entre 380 miembros de la Sociedad Chilena de Anestesia, representando un $23,4 \%$ de la población.

Las respuestas a las preguntas formuladas se describen a continuación:

¿Conoce la declaración de Helsinki sobre la seguridad del paciente en anestesia, firmada el año 2010? Un $30 \%$ de los encuestados señala conocerla, un $34 \%$ señala no conocerla y un $28 \%$ la conoce parcialmente.

En la declaración de Helsinki se señala la necesidad de establecer los protocolos descritos en la Tabla 1. De acuerdo a los centros asistenciales en donde trabajan los anestesiólogos encuestados se obtuvieron los resultados descritos en la tabla. De esta manera, se observa claramente como la evaluación preoperatoria está establecida en los centros en los cuales se desempeñan quienes contestaron la encuesta.

Aquí se observa como los protocolos que se encuentran presentes en un mayor porcentaje en los servicios de anestesia son control de infecciones (78\%) Hipertermia maligna (72\%) y control de fármacos (70\%). Por otra parte, los protocolos de hemorragia masiva, intoxicación por anestésicos locales y anafilaxia son los que se encuentran ausentes en un mayor porcentaje.

Al preguntar sobre la elaboración de un informe anual con las medidas tomadas y los resultados obtenidos en la mejora de la seguridad del paciente, nos encontramos que un $23 \%$ de los encuestados refiere que en sus centros de anestesia este informe es elaborado.

Con respecto a la elaboración de un informe anual sobre mortalidad y morbilidad de los pacientes, un $35 \%$ de los encuestados afirma que se realiza.

Además de los protocolos anteriormente mencionados, se consultó a los encuestados acerca de la existencia de un sistema de notificación de incidentes en sus centros, en este ítem se registró un $69 \%$ de respuestas afirmativas.

\section{Discusión}

De 100 invitaciones a contestar esta encuesta, llama la atención el alto porcentaje de respuesta que se obtuvo, alcanzando un $89 \%$ de los invitados a participar. Al parecer la seguridad del paciente en pabellón es un tema importante entre los anestesiólogos de la $\mathrm{SACH}$. Se observa en sus respuestas que existe en un 
alto porcentaje de protocolos activos en los servicios de anestesia para el control de infecciones (78\%), para el manejo de la hipertermia maligna $(72 \%)$ y el control de fármacos (70\%). No obstante, es llamativo el desconocimiento de otros protocolos de seguridad recomendados por la declaración de Helsinki y que son de gran importancia clínica para la seguridad del paciente, como son la hemorragia masiva, intoxicación por anestésicos locales, anafilaxia y cuidado perioperatorio del dolor. Aún más, algunos de estos protocolos están presentes como recomendaciones clínicas de la Sociedad Chilena de Anestesia.

Los entrevistados señalan la existencia de informes anuales de mortalidad y morbilidad en sus lugares de trabajo en el $39 \%$ de las encuestas, y sólo un $26 \%$ informa de medidas tomadas para la mejora de la seguridad de los pacientes.

En relación al alto porcentaje de existencia de protocolos de evaluación preoperatoria y de sistema de notificaciones, es necesario recordar que por ley el MINSAL considera alguno de ellos obligatorios para la acreditación de instituciones de salud pública o privada. Así, la Ley 19.337, marco legal para la acreditación de instituciones de salud, plantea, "Establecer los estándares mínimos que deberán cumplir los prestadores institucionales de salud... con el objetivo de garantizar que las prestaciones alcancen la calidad requerida para la seguridad de los usuarios. Deberá fijar estándares respecto de condiciones sanitarias, seguridad de instalaciones y equipos, aplicación de técnicas y tecnologías, cumplimiento de protocolos de atención, competencias de los recursos humanos, y en toda otra materia que incide en la seguridad de las prestaciones". Este sistema de acreditación, que se está aplicando tanto a instituciones públicas como privadas, crea un compromiso de aplicación de indicadores de seguridad, como la evaluación pre-anestésica, entre otros.

Sin embargo, en relación a las notificaciones de eventos adversos, la acreditación sólo hace exigible un sistema de notificación local, no existiendo un sistema nacional de comunicación entre hospitales y una estadística nacional de eventos adversos. Desconocemos si estos registros se realizan, tampoco cuales son los eventos registrados y si el registro es de forma electrónica o física, ya que solo los eventos centinelas, es decir de daño grave o muerte, son de notificación obligatoria.

La SACH, a través del comité de "Seguridad y Anestesia" ha priorizado como tema principal de su congreso anual en los años 2016 y 2017 "la seguridad del paciente en pabellón". Además, ha establecido un convenio de asociatividad con el sistema español de notificación en seguridad en anestesia y reanimación (SENSAR) permitiendo así la implementación y disponibilidad de un sistema de notificación nacional, voluntario y anónimo de incidentes y eventos adversos. Ha iniciado un plan de capacitación de anestesiólogos y enfermeras de pabellón y recuperación, y ha desarrollado una base de datos nacional que permitirá compartir online los incidentes tanto con hospitales nacionales adscritos como con centros españoles. Ya se han notificado más de 150 incidentes y se han propuesto más de 250 medidas de mejora a la fecha de presentación de este artículo.

Durante el año 2017 y 2018 SACH junto al comité de seguridad, trabajará en la puesta en marcha de talleres y formación de grupos locales destinados a la implementación y capacitación de la Declaración de Helsinki, además de elaborar recomendaciones generales tendientes a elevar el grado de seguridad del paciente.

\section{Conclusión}

Este trabajo es el primer esfuerzo por describir el interés de los anestesiólogos y en alguna medida el estado actual de la seguridad en pabellón en nuestro país, en cuanto a la existencia de los distintos protocolos establecidos como obligatorios por la Declaración de Helsinki. De acuerdo a lo expuesto, se hace imperioso fortalecer un sistema nacional de registro, desarrollar una política de seguridad en el perioperatorio y evaluar el impacto de estas acciones para corregir conductas, y de esta forma fortalecer la seguridad de nuestros pacientes todos los días. Una tarea ineludible de la SACH. 


\section{Referencias}

1. Castillo J. Sistemas de comunicación de incidentes en anestesia. Rev Esp Anestesiol Reanim. 2012;59:17-23.

2. Mellin-Olsen J, Staender S, Whitaker DK, Smith AF. The Helsinki declaration on patient safety in Anesthesiology. EJA. 2010;27:592-97.

3. Alexander Schleppers, Thomas Prien, Hugo Van Aken, Helsinki Declaration on patient safety in anaesthesiology: Putting words into practice - Experience in Ger- many, Best Practice \& Research Clinical Anaesthesiology, Volume 25, Issue 2, 2011, Pages 291304, ISSN 1521-6896.

4. Whitaker DK, Bratteb $\varnothing$ G, Smith AF, Staender SEA, The Helsinki Declaration on Patient Safety in Anaesthesiology: Putting words into practice. Best Pract Res Clin Anaesthesiol. 2011;25:277-290.

5. Guy Haller, Thierry Laroche, François Clergue, Morbidity in anaesthesia: Today and tomorrow, Best Practice \& Research Clinical Anaesthesiology, Volume 25, Issue 2, 2011, Pages 123-
132, ISSN 1521-6896.

6. Konstantinos Arfanis, Evridiki Fioratou, Andrew Smith, Safety culture in anaesthesiology: Basic concepts and practical application, Best Practice \& Research Clinical Anaesthesiology, Volume 25, Issue 2, 2011, Pages 229238, ISSN 1521-6896.

7. Monedero $\mathrm{P}$, Errando $\mathrm{CL}$, Adame M.M, Macías A.I, Garutti I; La Declaración de Helsinki sobre seguridad de los pacientes en anestesiología, Revista Española de Anestesiología y Reanimación, S0034-9356(13)70004-5. 